\title{
Numerical Simulation of the Dynamic Behaviors of a Gas Tungsten Welding Arc for Joining Magnesium Alloy AZ61A
}

\author{
Zhenmin $W A N G^{1)}$, Fang YAN ${ }^{2}$ and Pengcheng $Z H A O^{2) \dagger}$ \\ 1) School of Mechanical \& Automotive Engineering, South China University of Technology, Guangzhou 510640, China \\ 2) College of Electromechanical Engineering, Qingdao University of Science and Technology, Qingdao 266061, China \\ [Manuscript received 24 October 2012, in revised form 8 April 2013] \\ (c) The Chinese Society for Metals and Springer-Verlag Berlin Heidelberg
}

\begin{abstract}
Based on the Magneto-Hydro-Dynamic (MHD) theory, a united three-dimensional (3D) transient numerical model is developed to investigate the dynamic behaviors of arc plasma for a magnesium alloy AZ61A gas tungsten arc welding (GTAW) arc. The arc, electrode and workpiece are integrated into one calculation domain to avoid both presumed distribution of the current density at the electrode tip and the assumption of constant conditions of interface between welding arc and workpiece. The distributions of electric potential, current density, magnetic flux density, electromagnetic force, velocity, temperature, and pressure of the arc plasma in the 3D space are analyzed by using the numerical model. Results indicate that the maximum gradient of the electric potential in the whole arc space exists around the electrode tip, where the electric current density, electromagnetic force, and temperature are also the maximum. However, maximum pressure is found at the velocity stagnation, which is above the workpiece. Comparison between predicted temperature and measured one in arc region shows a good agreement.
\end{abstract}

\section{KEY WORDS: GTAW; Arc; MHD theory; Dynamic behavior; Numerical simulation}

\section{Introduction}

Magnesium alloys are becoming the preferred engineering material in automobile industry for its supreme specific strength, good machinability and easy recyclability ${ }^{[1]}$. Among the joining methods of magnesium alloys, the gas tungsten arc welding (GTAW) finds a wide application due to its advantages of economy, its high welding quality, and its steady arc length ${ }^{[2,3]}$.

During a magnesium alloy GTAW process, the welding arc exerts the heat flux, current density and pressure distribution on weld pool surface, which has a tight connection with thermal efficiency and melting efficiency of base metal. The arc also plays an important role in weld configuration and welding quality. Thus, it is of great significance to learn and understand the heat transfer and fluid flow of the arc

† Corresponding author. Assoc. Prof., Ph.D.; Tel.: +86 532 88956068; E-mail address: pczhao@qust.edu.cn (Pengcheng $\mathrm{ZHAO})$

DOI: $10.1007 / \mathrm{s} 40195-012-0187-0$ before the realization of the control of the quality and reliability of the weld. Therefore, the study of dynamic behavior of the welding arc lays a solid foundation for investigating the thermal process of the magnesium alloy GTAW process.

Great efforts have been made in getting dynamic information of welding arc by numerical simulation $^{[4-12]}$. Early researches ${ }^{[4-6]}$ have focused on the heat transfer and fluid flow in the arcs, but the influences of the welding materials are ignored. Studies on GTAW of steel or other metals are carried out concerning surface depressions of the weld pool and the interaction between arc and weld pool ${ }^{[7-12]}$. Moreover, some sophisticated calculations on the electric field, magnetic field, temperature field, and flow field of the arc and/or weld pool are reported ${ }^{[13,14]}$. Generally, the invariant Gaussian assumption for the heat flux, current density, and the arc pressure at the workpiece surface is employed when modeling the weld pool dynamics ${ }^{[15,16]}$. Therefore, bidirectional interactions in transfer phenomena between arc and weld pool are separated, and an empirical distribution on interface between arc and weld pool is usually 
employed.

Most present arc models are two-dimensional that are suitable to simulate the stationary axisymmetric $\operatorname{arc}^{[17,18]}$, which could be developed only when the magnetic field is uniform and the distribution of magnetic induction line is symmetric. However, the actual moving arc is generally non-axisymmetric due to backing strip, clamp and other welding conditions. Even for the stationary arc, some external perturbations such as the external magnetic field may deflect the arc from its axial symmetry. Therefore, a threedimensional (3D) plasma arc model should be developed that integrate the electrode and workpiece with arc together to avoid assumptions of contact conditions among electrode, arc plasma and workpiece.

For the numerical analysis of GTAW for magnesium alloy, Liang et al. ${ }^{[19]}$ investigated the heat source in overlap welding AZ31B magnesium alloy by lasertungsten inert gas arc. However, there has little been reported on GTAW arc behavior so far.

The present paper aims to investigate its dynamic behaviors, including the electric potential, current density, magnetic field, electromagnetic force, velocity, temperature, and pressure of a magnesium alloy GTAW arc by using a united 3D transient numerical model developed.

\section{Model}

A GTAW process is schematically shown in Fig. 1. Direct current electrode positive (DCEP) connection is applied for the welding of magnesium alloy. During the welding, a direct current high-voltage between the anode (electrode) and the cathode (workpiece) causes ionization of argon. The passage of an electric current through the ionized plasma region generates a self-induced magnetic field. Thus the magnetic force is excited by the interaction between current and magnetic field. Under the action of the electromagnetic force, the plasma is accelerated toward the cathode in a form of characteristic cathode jet. Due to the electrical resistance of the plasma, the heat energy produced by the current maintains the plasma in the ionized state and provides the heating mechanism for the welding process.

Some assumptions are made in the model to simplify the model as follows. (1) The arc is in local thermal equilibrium (LTE), which means that the temperature of both electron and heavy particle are the same. (2) The plasma is optically thin so that radiation may be accounted for using an optically thin radiation loss per unit volume. (3) The arc plasma consists of pure argon at atmospheric pressure. (4) Buoyancy and the heating effect of viscous dissipation are negligible. (5) The plasma flow is laminar and incompressible.

\subsection{Governing equations}

The welding arc consists of a flow of electrical

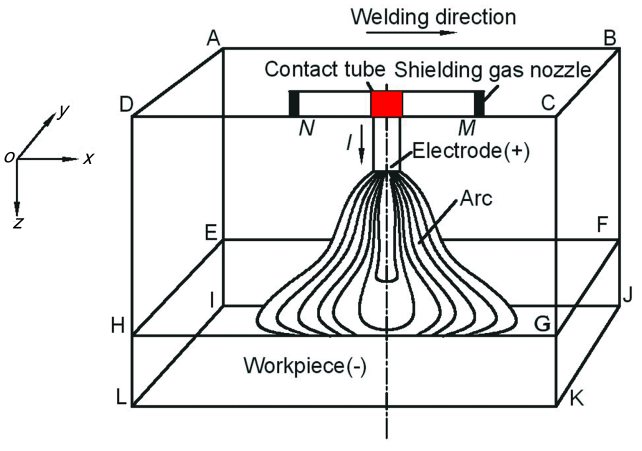

Fig. 1 Schematic of GTAW operation in Cartesian coordinate system

charged particles, which could be analyzed by a combined model of fluid mechanics and electromagnetic, and satisfies the Magneto-Hydro-Dynamics (MHD) theory. In Cartesian coordinate system, the governing equations for the plasma arc may be written as follows.

\subsubsection{Basic governing equations}

Mass continuity equation

$$
\frac{\partial U}{\partial x}+\frac{\partial V}{\partial y}+\frac{\partial W}{\partial z}=0
$$

Momentum equations

$$
x \text {-momentum }
$$

$$
\begin{gathered}
\frac{\partial(\rho u)}{\partial t}+\nabla \cdot(\rho \vec{V} u)=-\frac{\partial P}{\partial x}+\mu\left(\frac{\partial^{2} u}{\partial x^{2}}+\frac{\partial^{2} u}{\partial y^{2}}+\frac{\partial^{2} u}{\partial z^{2}}\right)+ \\
J_{y} B_{z}-J_{z} B_{y}
\end{gathered}
$$

$y$-momentum

$$
\begin{gathered}
\frac{\partial(\rho v)}{\partial t}+\nabla \cdot(\rho \vec{V} v)= \\
J_{z} B_{x}-J_{x} B_{z}
\end{gathered}
$$

$z$-momentum

$$
\begin{gathered}
\frac{\partial(\rho w)}{\partial t}+\nabla \cdot(\rho \vec{V} w)=-\frac{\partial P}{\partial z}+\mu\left(\frac{\partial^{2} w}{\partial x^{2}}+\frac{\partial^{2} w}{\partial y^{2}}+\frac{\partial^{2} w}{\partial z^{2}}\right)+ \\
J_{x} B_{y}-J_{y} B_{x}
\end{gathered}
$$

Energy equation

$$
\begin{aligned}
\frac{\partial(\rho h)}{\partial t}+\nabla & (\rho \vec{V} h)=\nabla \cdot\left(\frac{k}{C_{p}} \nabla h\right)+\frac{J_{x}^{2}+J_{y}^{2}+J_{z}^{2}}{\sigma_{e}}-S_{R}+ \\
& \frac{5 k_{b}}{2 e}\left(\frac{J_{x}}{C_{p}} \frac{\partial h}{\partial x}+\frac{J_{y}}{C_{p}} \frac{\partial h}{\partial y}+\frac{J_{z}}{C_{p}} \frac{\partial h}{\partial z}\right)
\end{aligned}
$$

\subsubsection{Maxwell's equations}

However, there are electromagnetic force components $J_{y} B_{z}-J_{z} B_{y}, J_{z} B_{x}-J_{x} B_{z}$, and $J_{x} B_{y}-J_{y} B_{x}$ in the Eqs. (2)-(4), and the energy equation contains the Joule-heat term and an additional term which represents the transport of electron enthalpy due to the drift of electrons. Thus, it is necessary to solve Maxwell's equations for the electromagnetic field. 
Current continuity equation

$$
\frac{\partial}{\partial x}\left(\sigma_{e} \frac{\partial \phi}{\partial x}\right)+\frac{\partial}{\partial y}\left(\sigma_{e} \frac{\partial \phi}{\partial y}\right)+\frac{\partial}{\partial z}\left(\sigma_{e} \frac{\partial \phi}{\partial z}\right)=0
$$

Ohm's law

$$
J_{x}=-\sigma_{e} \frac{\partial \phi}{\partial x}, \quad J_{y}=-\sigma_{e} \frac{\partial \phi}{\partial y}, \quad J_{z}=-\sigma_{e} \frac{\partial \phi}{\partial z}
$$

Poisson equation

$$
\begin{aligned}
& \frac{\partial^{2} B_{x}}{\partial x^{2}}+\frac{\partial^{2} B_{x}}{\partial y^{2}}+\frac{\partial^{2} B_{x}}{\partial z^{2}}=-\mu_{0}\left(\frac{\partial J_{z}}{\partial y}-\frac{\partial J_{y}}{\partial z}\right) \\
& \frac{\partial^{2} B_{y}}{\partial x^{2}}+\frac{\partial^{2} B_{y}}{\partial y^{2}}+\frac{\partial^{2} B_{y}}{\partial z^{2}}=-\mu_{0}\left(\frac{\partial J_{x}}{\partial z}-\frac{\partial J_{z}}{\partial x}\right) \\
& \frac{\partial^{2} B_{z}}{\partial x^{2}}+\frac{\partial^{2} B_{z}}{\partial y^{2}}+\frac{\partial^{2} B_{z}}{\partial z^{2}}=-\mu_{0}\left(\frac{\partial J_{y}}{\partial x}-\frac{\partial J_{x}}{\partial y}\right)
\end{aligned}
$$

In Eqs. (1)-(10), $\rho$ is the density, $t$ is the time, $u$, $v$ and $w$ are, respectively, the velocity components in the $x, y$, and $z$ directions, $\vec{V}$ is velocity vector, $P$ is the pressure, $h$ is the enthalpy, $k$ is the thermal conductivity, $C_{p}$ is the specific heat at constant pressure, $\mu$ is the viscosity, $\sigma_{\mathrm{e}}$ is the electric conductivity, $k_{\mathrm{b}}$ is the Stefan-Boltzmann constant, $e$ is the elementary charge, $J_{x}, J_{y}$, and $J_{z}$ are the current density components in the $x, y$, and $z$ directions, respectively, and $B_{x}, B_{y}$, and $B_{z}$ are the magnetic field components in the $x, y$, and $z$ directions, respectively, $\phi$ is the electric potential, $\mu_{0}$ is the magnetic permeability of vacuum. The last two terms in the momentum Eqs. (2)-(4) represent the respective components of the electromagnetic forces. The last three terms in Eq. (5) are the Joule heat, radiation loss, and electron enthalpy flow, respectively.

\subsection{Calculation domains and processing of solid-gas interface}

It is important to choose reasonable calculation domains in simulating object. Generally speaking, the chosen calculation domains should be as small as possible under a prerequisite of being able to satisfy the simulated work and should make it easier to define boundary conditions.

The computational domains for the welding arc are also shown in Fig. 1, which includes an anode region, an arc region, and a cathode region. The domain is symmetric in $y$ direction, the plane CDLK is as the symmetric plane, so only a half of the space in the domains is considered in order to reduce the computational burden. The electrode is added into the electromagnetic calculation domain which avoids the assumption of current density distribution at the tip of the electrode. Therefore the current density can be obtained by calculating rather than assumption. The workpiece region is included in the calculation domain too, which not only avoid the assumption of the boundary conditions and deformation interface between welding arc and workpiece, but also realize the two-way coupling between the welding arc and the weld pool.

However, because of the characteristics of GTAW, the electrode does not melt and locates in the middle of the calculation domains when calculating flow field for GTAW arc, which makes the numerical study on flow field become a complex interaction between the solid and gas. An integer solving method is used to deal with such problems ${ }^{[20]}$, so the coupling interface becomes the interior of the calculation region, and meets the continuity principle on the interface when the governing equations are discretized. The velocity in the electrode is obtained by using viscosity coefficient method to ensure the velocity is zero, while the actual viscosity of argon is given to the arc plasma to obtain the velocity of arc plasma.

\subsection{Boundary conditions}

The study on dynamic behavior of the arc includes the prediction of the electric field, magnetic field, flow field, temperature field, and pressure field, so that the boundary conditions must be listed respectively. Along the symmetric plane CDLK, symmetry conditions are used.

\subsubsection{Electric field boundary conditions}

The boundary conditions for the electric field are shown in Table 1. Electrical conductivity of cathode is $6.5 \times 10^{6} \mathrm{~s} / \mathrm{m}$, which is greater than the electrical conductivity of plasma arc, so the cathode is assumed to be a perfect conductor relative to the plasma. Thus, the bottom plane of the workpiece IJKL is taken to be isopotential and the electrical potential is defined to be zero. The top plane ABCD is the most important boundary conditions in electric field, where the welding current is assumed to be uniformly distributed along $z$ direction when it flows into the electrode. The gradient of electric potential for the electrode boundary is determined as

$$
-\sigma_{\mathrm{e}} \frac{\partial \phi}{\partial z}=\frac{I}{\pi R_{\mathrm{a}}^{2}}\left(r<R_{\mathrm{a}}\right)
$$

where $r=\sqrt{x^{2}+y^{2}}, R_{\mathrm{a}}$ is the radius of the electrode, and $I$ is the welding current.

Zero gradients for the electric potential in vertical direction have been set on the top boundary $\left(r>R_{\mathrm{a}}\right)$. The symmetric plane and all side boundary planes represent the condition that no current flow crosses these boundaries.

\subsubsection{Magnetic field boundary conditions}

(1) If $r>R_{\mathrm{a}}$, the circular curve $L$ is selected as an Ampere loop, which is passing the point $P$ and the radius is $r$, the plane where Ampere loop located in is perpendicular to the axis. According to Ampere's 
Table 1 Boundary conditions for electric potential and magnetic field equations in different planes



law, the following is true:

$$
\oint_{\mathrm{L}} \vec{B} \cdot \mathrm{d} l=B \oint_{\mathrm{L}} \mathrm{d} l=B 2 \pi r=\mu_{0} I
$$

The self-induced magnetic field $B$ is calculated as follows:

$$
B=\frac{\mu_{0} I}{2 \pi r}
$$

Eq. (12) shows the self-induced magnetic field $B$ is inversely proportional to the $r$.

(2) If $r<R_{\mathrm{a}}$, the circular curve $L$ is similarly selected as an Ampere loop, which is passing the point $P^{\prime}$ and the radius is $r$, as shown in Fig. 2. The difference between this case and above case is that the current surrounded by the loop $L$ is not all the current in the electrode, but part of it, as follows:

$$
I^{\prime}=\frac{I}{\pi R_{\mathrm{a}}^{2}} \pi r^{2}=\frac{r^{2}}{R_{\mathrm{a}}^{2}} I
$$

According to Ampere's law, the following is true:

$$
\oint_{L} \vec{B} \cdot \mathrm{d} l=B \oint_{L} \mathrm{~d} l=B \cdot 2 \pi r=\mu_{0} I^{\prime}=\mu_{0} \frac{r^{2}}{R_{\mathrm{a}}^{2}} I
$$

At last, the self-induced magnetic field $B$ is determined by

$$
B=\frac{\mu_{0} I}{2 \pi R_{\mathrm{a}}^{2}}
$$

Eq. (13) shows the self-induced magnetic field $B$ is proportional to the $r$.

(3) At central axis $(r=0), B=0$. $\frac{\mu_{0} I}{2 \pi R_{\mathrm{a}}}$.

(4) At the surface of the electrode $\left(r=R_{\mathrm{a}}\right), B=$

The projected $x, y$, and $z$ components are determined as follows:

$$
B_{x}=\frac{y}{r} B, B_{y}=-\frac{x}{r} B, B_{z}=0
$$

For the symmetric plane, the component of the magnetic field in $x$ direction is zero, and the gradients of both magnetic field $\partial B_{y} / \partial y$ and $\partial B_{z} / \partial y$ are zero. For all the side planes and the bottom plane, the gradient of the magnetic field is simply assumed to be zero, as shown in Table 1.

\subsubsection{Energy boundary conditions}

An adiabatic condition is specified on the symmetric plane, which is $\partial T / \partial y=0$. At the other boundary surfaces, a temperature is taken as $1000 \mathrm{~K}$, which is

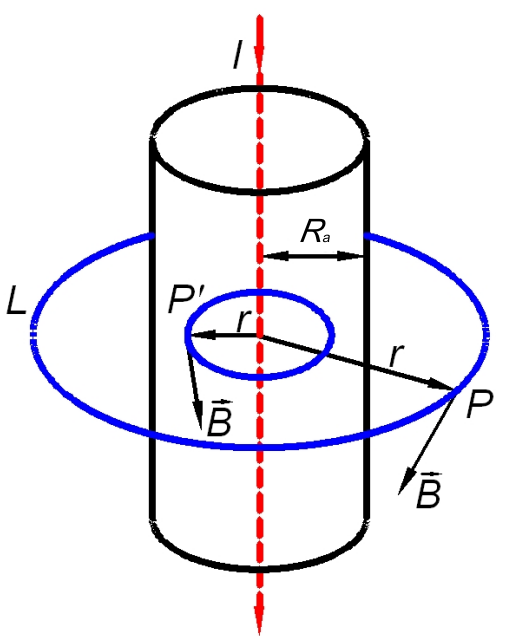

Fig. 2 The magnetic field of an infinite cylinder

convenient for dealing with boundary conditions. Because the temperature of those boundary surfaces is much lower than that of plasma arc and those boundary surfaces nearby occupy little enthalpy, so they have little effect on calculating results.

\subsubsection{Momentum boundary conditions}

The momentum boundary conditions used are given in Table 2. The imposed shielding gas flow is set through a boundary condition on $W$ along $M N$. For the inflow of gas from the nozzle, the velocity components in $x$ and $y$ directions can be neglected, because the ionized gas flow velocity here is relatively small compared with the arc flow velocity, and its distribution hardly affect the final characters of the plasma arc. The velocity component in $z$ direction is determined from the formula of pipe flow is as follows.

$$
W=\frac{2 Q}{\pi \rho} \frac{\left[R_{n}^{2}-r^{2}+\left(R_{n}^{2}-R_{a}^{2}\right) \frac{\ln \left(r / R_{n}\right)}{\ln \left(R_{n} / R_{a}\right.}\right]}{\left[R_{n}^{4}-R_{a}^{4}+\frac{\left(R_{n}^{2}-R_{a}^{2}\right)^{2}}{\ln \left(R_{n} / R_{a}\right)}\right]}
$$

where $Q$ is the inflow rate of shielding gas, and $R_{n}$ is the internal radius of the shielding nozzle. As to the area where $r>R_{n}, z$ direction gradient of $W$ is equal to zero.

The boundary conditions at the symmetric plane CDLK in the model is straightforward. The velocity components in $y$ direction are zero, while the gradients of velocity $\partial U / \partial y$ and $\partial W / \partial y$ are also zero. Zero velocities are specified along the bottom plane of the workpiece. For all the side planes, the gradients of velocity are zero. 
Table 2 Momentum boundary conditions in different planes

\begin{tabular}{ccccccc}
\hline & ABCD & IJKL & ADLI & BCKJ & ABJI & CDLK \\
\hline$u$ & 0 & 0 & $\frac{\partial u}{\partial x}=0$ & $\frac{\partial u}{\partial x}=0$ & $\frac{\partial u}{\partial y}=0$ & $\frac{\partial u}{\partial y}=0$ \\
$v$ & 0 & 0 & $\frac{\partial v}{\partial x}=0$ & $\frac{\partial v}{\partial x}=0$ & $\frac{\partial v}{\partial y}=0$ & 0 \\
$w$ & Eqs. $(2)-(15), r<R_{n} ; \frac{\partial w}{\partial z}=0, r>R_{n}$ & 0 & $\frac{\partial w}{\partial x}=0$ & $\frac{\partial w}{\partial x}=0$ & $\frac{\partial w}{\partial y}=0$ & $\frac{\partial w}{\partial y}=0$ \\
\hline
\end{tabular}

Table 3 Welding parameters

\begin{tabular}{ccc}
\hline Nomenclature & Symbol & Value (unit) \\
\hline Welding current & $I$ & $110(\mathrm{~A})$ \\
Voltage & $V$ & $24(\mathrm{~V})$ \\
Shielding gas flow rate & $Q$ & $8\left(\mathrm{~L} \cdot \mathrm{min}^{-1}\right)$ \\
Welding speed & $v$ & $0.6\left(\mathrm{~m} \cdot \mathrm{min}^{-1}\right)$ \\
Arc length & $L$ & $9(\mathrm{~mm})$ \\
Diameter of shielding gas nozzle & $D_{\mathrm{N}}$ & $8(\mathrm{~mm})$ \\
Electrode radius & $R_{\mathrm{a}}$ & $1.6(\mathrm{~mm})$ \\
Stem length of electrode & $\delta$ & $15(\mathrm{~mm})$ \\
\hline
\end{tabular}

\subsubsection{Pressure field boundary conditions}

An atmospheric pressure is imposed at the inlet and outlet, which means that the pressure there is set to be $1.013 \times 10^{5} \mathrm{~Pa}$. For the bottom plane of the workpiece and the symmetric plane, the gradient of pressure field is simply assumed to be zero.

\section{Numerical Simulation}

\subsection{Numerical method}

The control volume integration method is utilized to discretize the governing equations, and the SIMPLE algorithm ${ }^{[21]}$ is employed to solve the strongly coupling nonlinear algebraic equations. The numerical iteration procedure is as follows:

(1) Grid generation. The calculation domains as shown in Fig. 1 are discretized by using the inner node method. In order to enhance the accuracy of calculation, a non uniform grid system is adopted. Finer grid sizes are divided near the arc center, in which the size of meshes is set as $0.2 \mathrm{~mm}$, for the temperature gradient in the central area of the arc is higher than that of other parts. Non uniform meshes are employed in the region away from the arc center, and the expansion coefficient is 1.2 .

(2) Electric field calculation. The plasma arc is defined as the charged fluid, and its electrical conductivity is strongly temperature dependent. The conductivity of argon is zero when the temperature is under $3000 \mathrm{~K}$, only when the temperature increases above $5000 \mathrm{~K}$ that the argon begins to conduct electricity, for the ionizing and conducting temperature of which reaches almost $1000 \mathrm{~K}$. So an initial temperature of $10000 \mathrm{~K}$ is specified in calculating electric field. At last, the current continuity equation and Ohm's law are solved in the whole domain. The distribution of potential, current density, and Ohmic heating are then calculated.

(3) Calculation of the self-induced magnetic field.
The magnetic field (Eqs. (8)-(10)) are solved in the whole domain for the values of $B_{x}, B_{y}, B_{z}$ and the electromagnetic forces are then calculated for the momentum equations.

(4) Calculation of the flow field and pressure field. Eqs. (1)-(4) and the associated boundary conditions are solved iteratively to obtain the distributions of velocity and pressure of the arc plasma.

(5) Calculation of the temperature field. Eq. (5) is solved to get the new temperature distribution. Thermal physical properties of the arc plasma are updated. Return to step (2) and this loop is repeated until the convergence is achieved.

(6) Data output. The calculation results are written to the specified files, and the program advances to the next time step to continue calculating.

\subsection{The properties of workpiece and thermo-physical properties of gas}

The workpiece in present study is an AZ61A magnesium alloy plate with a size of $20 \mathrm{~mm} \times$ $18 \mathrm{~mm} \times 3 \mathrm{~mm}$. The tungsten electrode is employed and pure argon is used as shielding gas. Welding parameters are shown in Table 3, while thermo-physical properties of the magnesium alloy ${ }^{[22]}$ are listed in Table 4 .

Thermo-physical properties of the shielding gas, including density $\rho$, enthalpy $h$, thermal conductivity $k$, specific heat $C_{\mathrm{p}}$, viscosity $\mu$, electric conductivity $\sigma_{\mathrm{e}}$, and radiation losses $S_{\mathrm{R}}$ are strongly temperature dependent and their relation with temperature are plotted in Ref. [20].

\section{Results and Discussion}

\subsection{The electric field}

Fig. 3 shows the electric potential distribution at different surfaces at $t=0.5 \mathrm{~s}$. The workpiece is under the marked red lines. As shown in Fig. 3, the maximum electric potential occurs in the electrode region while the electric potential of the workpiece is zero, 
Table 4 Physical parameters used in the model ${ }^{[22]}$

\begin{tabular}{ccc}
\hline Nomenclature & Symbol & Value (unit) \\
\hline Electrical conductivity & $\sigma_{\mathrm{e}}$ & $6.5 \times 10^{6}\left(\Omega^{-1} \cdot \mathrm{m}^{-1}\right)$ \\
Electric charge & $e$ & $1.602 \times 10^{-19}(\mathrm{C})$ \\
Acceleration of gravity & $\mathrm{g}$ & $9.8\left(\mathrm{~m} \cdot \mathrm{s}^{-2}\right)$ \\
Stefan-Boltzmann constant & $k_{\mathrm{b}}$ & $1.38 \times 10^{-23}\left(\mathrm{~J} \cdot \mathrm{K}^{-1}\right)$ \\
Environment temperature & $T_{0}$ & $300(\mathrm{~K})$ \\
Melting point & $T_{\mathrm{m}}$ & $880(\mathrm{~K})$ \\
Magnetic permeability of vacuum & $\mu_{0}$ & $1.25663706 \times 10^{-6}\left(\mathrm{~N} \cdot \mathrm{A}^{-2}\right)$ \\
Heat transfer coefficient & $h_{\mathrm{c}}$ & $80\left(\mathrm{~W} \cdot \mathrm{m}^{-2} \cdot \mathrm{K}^{-1}\right)$ \\
\hline
\end{tabular}
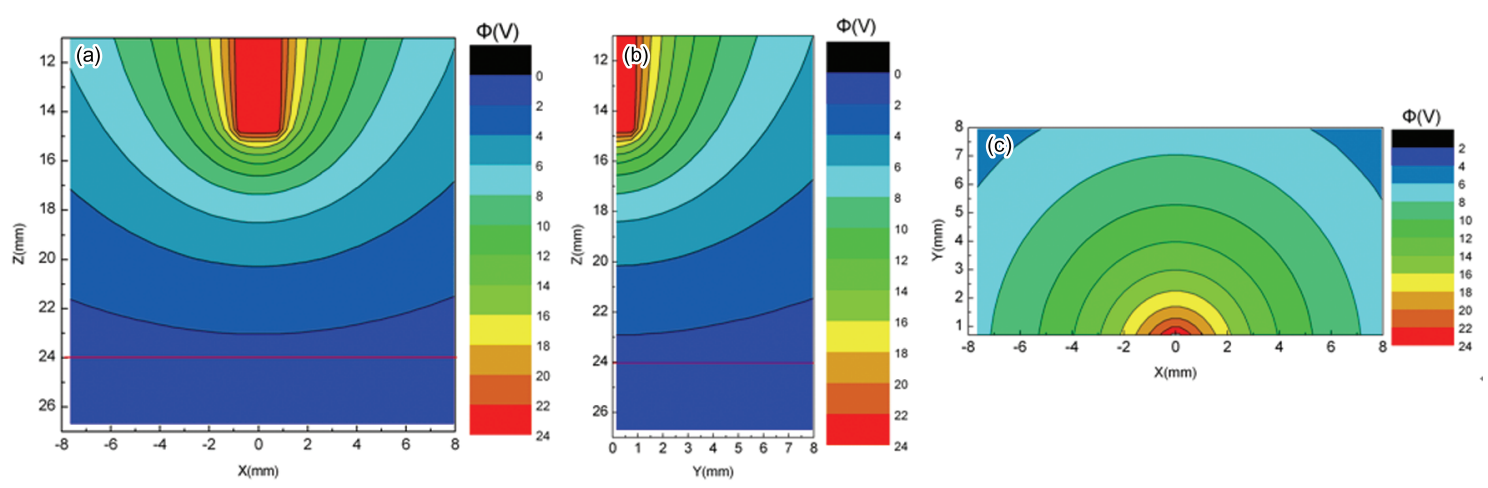

Fig. 3 Distribution of electrical potential at $t=0.5 \mathrm{~s}$ : (a) side view; (b) front view; (c) top view

because the welding condition is DCEP. The electric potential in the arc decreases rapidly with increasing distance from the anode. The electric potential (voltage) drop is $15.4 \mathrm{~V}$ from the electrode to the workpiece. It can also be found that the electrical potential contours are denser around the electrode tip but diverges gradually with increasing distance from the electrode, which demonstrates that the electric potential drop is larger around the electrode tip than other regions.

Fig. 4 indicates the centerline distribution of the electrical potential at $t=0.5 \mathrm{~s}$. In the electrode region, the electric potential does not change, with a constant electric potential of $24 \mathrm{~V}$. However, the electric potential deceases sharply in the arc region. A steep gradient of the electric potential is found at $1 \mathrm{~mm}$ below the electrode tip (from $15 \mathrm{~mm}$ to $16 \mathrm{~mm}$ in $z$ direc-

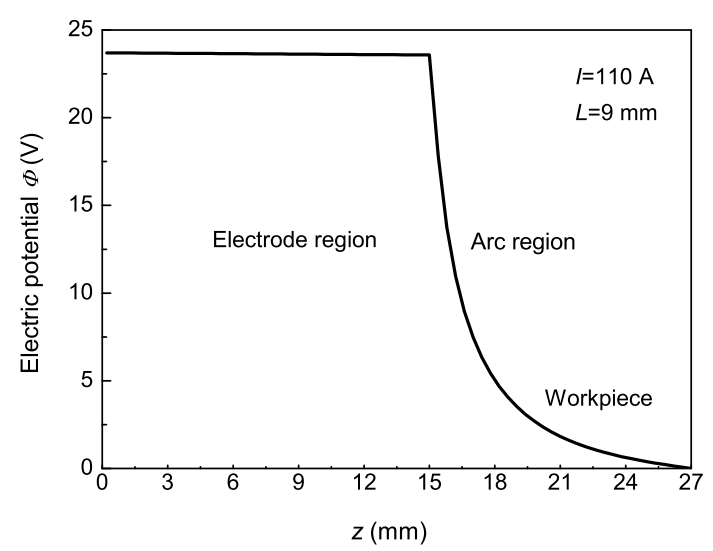

Fig. 4 Electrical potential along symmetric axis at $t=0.5 \mathrm{~s}$ tion), where the electric potential varies from $23.9 \mathrm{~V}$ to $12.8 \mathrm{~V}$. Because the temperature around the electrode surface is relatively low, there is a thin layer of cold air, which results in a lower electrical conductivity and then generates a greater voltage drop. Beyond this region, the gradient of the electric potential changes slowly. At the bottom side of the workpiece, the electric potential is zero.

The current density vector fields at different surfaces at $t=0.5 \mathrm{~s}$ are shown in Fig. 5 . The direction of the arrow represents the flow direction of the welding current while the length of the arrow represents the values of the current density. It can be seen that the current density $J_{z}$ remains constant in the electrode region, which is $5.47 \times 10^{7} \mathrm{~A} / \mathrm{m}^{2}$ in current study. However, the distribution of the current density $J_{z}$ is not uniform in the arc region. The current density achieves its greatest value at the tip of the electrode, and it decreases rapidly with increasing distance from the electrode. Fig. 5(c) shows current diverges from the electrode center.

Fig. 6 shows the calculated current density $J_{z}$ in the arc region at $x$ direction on the symmetric plane at $t=0.5 \mathrm{~s}$. Under current welding conditions, the maximum values of the current density are obtained at the electrode tip, and then it decrease gradually with increasing distance from the $z$ axis. Since the electrical conductivity depends on the temperature, the current density and the temperature influence each other. Thus the temperature determines the distribution of the electrical conductivity, which finally affects the current density distribution. At the same time, the current density distribution determines the Joule heat, which has an impact on the temperature 

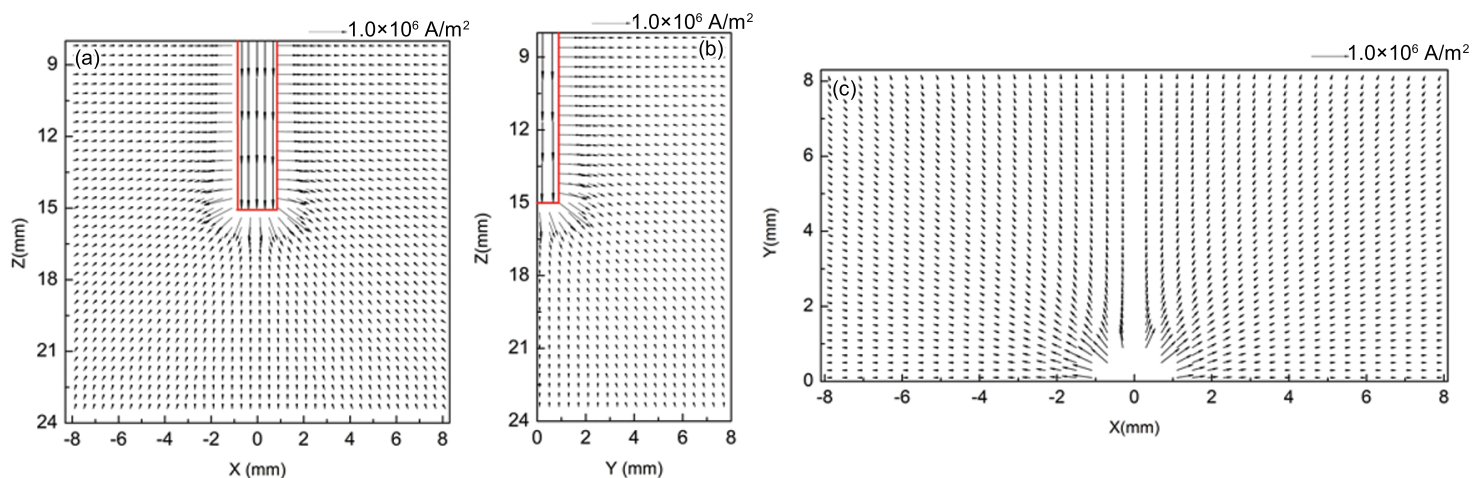

Fig. 5 Distribution of the current density vector at different sections at $t=0.5 \mathrm{~s}$ : (a) side view; (b) front view; (c) top view

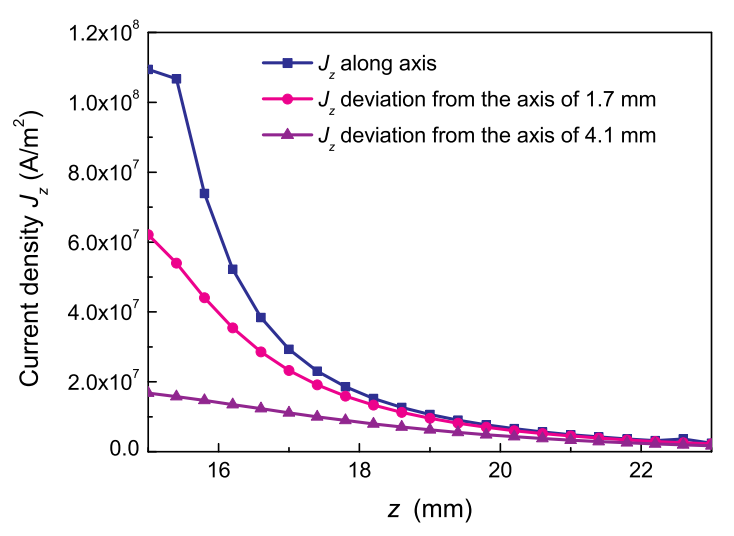

Fig. 6 Current density $J_{\mathrm{z}}$ in different region of arc on the symmetric plane at $t=0.5 \mathrm{~s}$

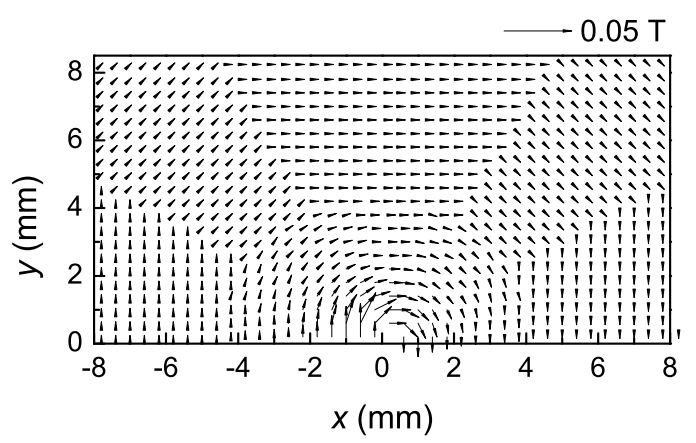

Fig. 7 Magnetic field vectors at $x o y$ section at $t=0.5 \mathrm{~s}$

distribution. There is more current flow along the arc center than through other region.

\subsection{The magnetic field}

According to Ampere's law, flowing electron would produce magnetic field. Fig. 7 shows the calculated magnetic field vectors at $x o y$ section at $t=0.5 \mathrm{~s}$. It can be seen that the magnetic induction line is a series of closed curves around the electrode. On the same circumference the magnetic intensity is the same, and its direction is on the tangent of the circumference. The direction of magnetic induction line and welding current is identical with right-hand rule. The magnetic induction lines are denser near the arc center than in other areas.
The vectors of electromagnetic force at $t=0.5 \mathrm{~s}$ are shown in Fig. 8. It is noted that the direction of the electromagnetic force is perpendicular to the $z$ axis in the electrode region, which presents two components in $x$ and $y$ directions. While in the plasma zone directions of these two components are inward and downward, respectively. Therefore the $x$ component will push the arc plasma moving downward and the $y$ component will constrict the arc. It is obvious that the effects of the electromagnetic force on the arc include constricting the arc from free expansion and pushing the arc plasma moving downward.

To further analyze the electromagnetic force distribution in the $3 \mathrm{D}$ arc space, the electromagnetic force components in $x, y$, and $z$ directions on the symmetric plane at $t=0.5 \mathrm{~s}$ is calculated respectively, as indicated in Fig. 9. Both components in $x$ and $y$ directions are negative and inward. They are smaller near the arc centre than that at other region. But at $z=15 \mathrm{~mm}$, they have their maximum values at 0.5 or $1.0 \mathrm{~mm}$ away from the $z$ axis, where $F_{x}$ is about $1.6 \times 10^{4} \mathrm{~N} / \mathrm{m}^{3}$ and $F_{y}$ is about $6 \times 10^{4} \mathrm{~N} / \mathrm{m}^{3}$, respectively. The reason is that the current density achieves its biggest value there. In addition, the electromagnetic force decreases with the increase of the distance to arc center. The distribution of the electromagnetic force in $z$ direction is different from that in $x$ and $y$ directions. It is noted that its value is positive, which indicates that it pushes the arc plasma moving downward. At $z=15 \mathrm{~mm}$ the electromagnetic force has the maximum value, about $8.5 \times 10^{3} \mathrm{~N} / \mathrm{m}^{3}$, and decreases along $z$ direction. The electromagnetic force reaches the minimum value at $z=23 \mathrm{~mm}$.

The calculated flow velocity of the plasma arc is shown in Fig. 10. It illustrates that the shielding gas and arc plasma flows down along $z$ axis under the influence of the electromagnetic force after flowing from the nozzle. The plasma flow finally reaches the workpiece surface and then leaves the arc domain from outer boundaries. As a result of the stagnation of the workpiece surface, the plasma gas flows radically outward along the workpiece surface.

Fig. 11 shows the distribution of the arc pressure and the velocity along its centerline. The plasma 

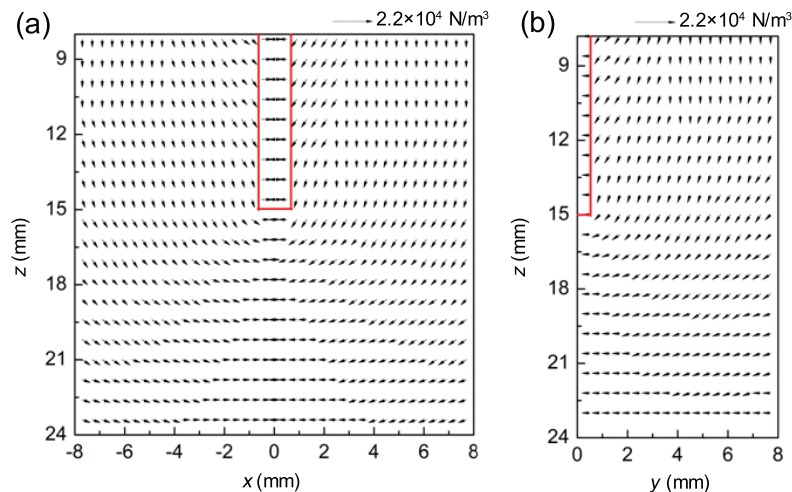

(c)

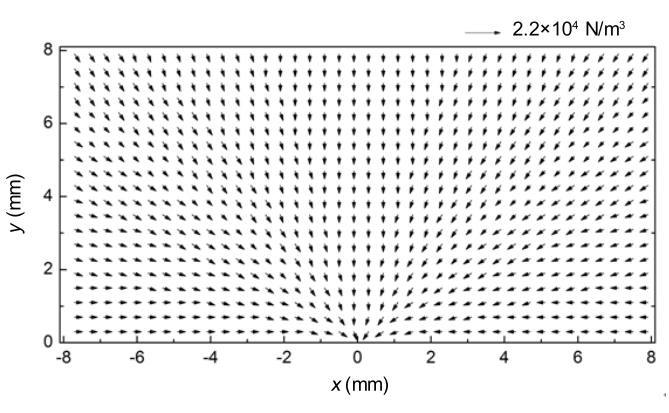

Fig. 8 Distribution of electromagnetic force at different sections at $t=0.5 \mathrm{~s}$ : (a) side view; (b) front view; (c) top view
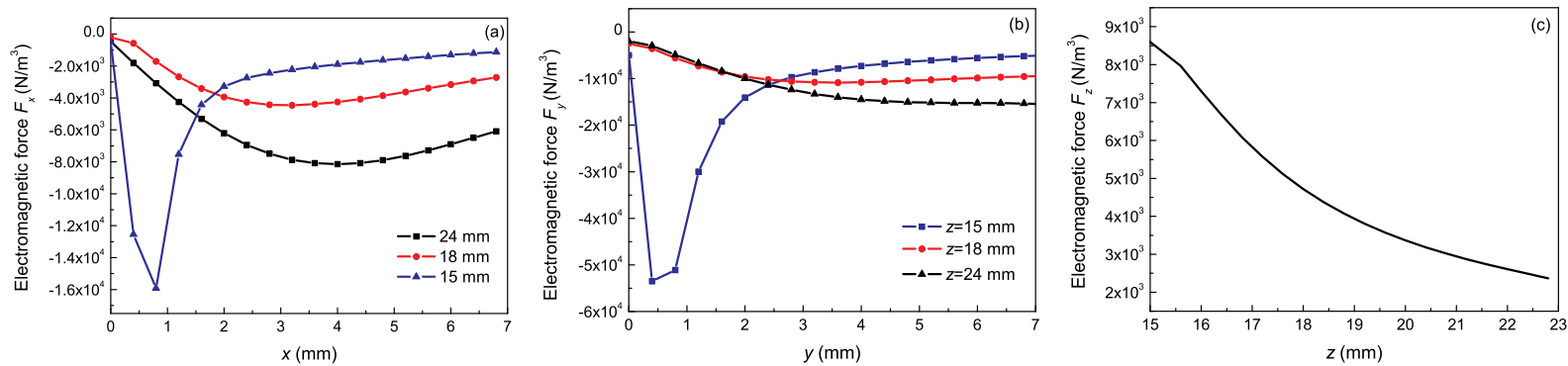

Fig. 9 The distribution of electromagnetic force at different direction on the symmetric plane at $t=0.5 \mathrm{~s}$ : (a) $x$ direction; (b) $y$ direction; (c) $z$ direction
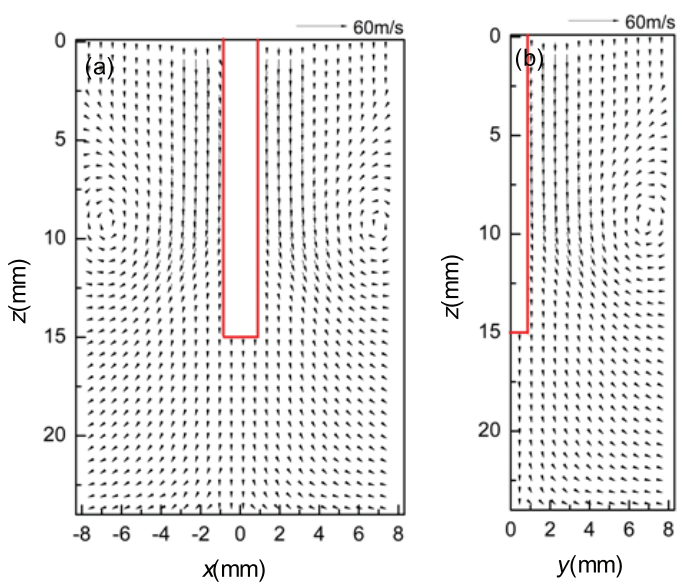

Fig. 10 Distribution of velocity vector at $t=0.5 \mathrm{~s}$ : (a) side view; (b) front view

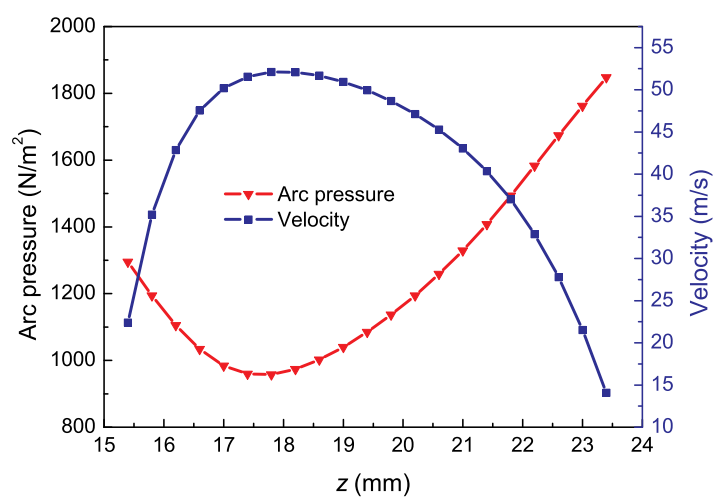

Fig. 11 The distribution of arc pressure and velocity along symmetric axis gas enters the arc with a low velocity, then the velocity of the plasma gas increases rapidly from electrode tip and the maximum value of $52 \mathrm{~m} / \mathrm{s}$ is obtained at $z=17.8 \mathrm{~mm}$. After then, the flow velocity decreases slightly in the middle of the arc column. As a result of the stagnation of the workpiece surface, the velocity decreases rapidly on the workpiece surface. However, the maximum pressure of $1848 \mathrm{~N} / \mathrm{m}^{2}$ is found at the velocity stagnation, which is above the workpiece $(z=23.4 \mathrm{~mm})$ because of the swash of arc plasma against the workpiece. The minimum arc pressure is $958 \mathrm{~N} / \mathrm{m}^{2}$ at $z=17.8 \mathrm{~mm}$, where the largest velocity occurs.

\subsection{The temperature field}

The simulated isotherms are shown in Fig. 12,

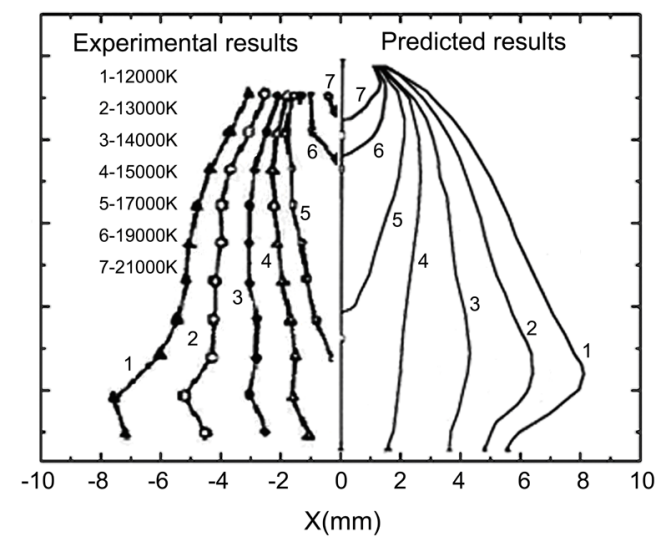

Fig. 12 Comparison of predicted and measured temperature field results of the arc 
where the temperature distribution measured by $\mathrm{Hsu}$ et al. ${ }^{[4]}$ using the spectrometry method is also given as a comparison. At first, the highest temperature of $21000 \mathrm{~K}$ occurs near the electrode tip, because the current density achieves its maximum value there. The isotherms diverge gradually with the expanding of arc fracture surface, and the typical bell shape plasma arc is clearly observed. Moreover, comparison between the predicted and the measured temperature distributions shows a fairly good agreement ${ }^{[4]}$.

\section{Conclusions}

(1) A 3D mathematical model based on the MHD theory has been developed to describe the transient heat transfer and fluid flow in GTAW welding of magnesium alloy AZ61A, which integrates the electrode and workpiece with arc together.

(2) The dynamic behaviors for GTAW arc are analyzed numerically. The calculated results show that the largest gradient of the electric potential in the whole arc space locates around the electrode tip, where there exists the maximum electric current density, electromagnetic force, and temperature. However, the highest pressure is found at the velocity stagnation, which is above the workpiece.

(3) Comparison between predicted temperature in arc regions and measured one in other study is carried out and the results show a good agreement.

\section{Acknowledgements}

This work was financially supported by the Fundamental Research Funds for the Central Universities (No. 2012ZZ0059), the National Natural Science Foundation of China (No. E51375173) and the Open Fund of State Key Laboratory of Materials Processing and Die \& Mould Technology (No. 2011-P02). Part of this work was also a project of Shandong Province Higher Educational Science and Technology Program (No. J12LA16).

\section{REFERENCES}

[1] Y. Oishi, N. Kawabe, A. Hoshima, Y. Okazaki and A. Kishimoto, SEI Technol. Rev. 56 (2003) 54.

[2] L. Liu and C. Dong, Mater. Lett. 60 (2006) 2194.

[3] A. Munitz, C. Cotler, A. Stern and G. Kohn, Mater. Sci. Eng. A 302 (2001) 68.

[4] K.C. Hsu, K. Etemadi and E. Pfender, J. Appl. Phys. 54 (1983) 1293.

[5] K.C. Hsu and E. Pfender, J. Appl. Phys. 54 (1983) 4359.

[6] J. McKelliget and J. Szekely, Metall. Trans. A 17 (1986) 1139.

[7] S.Y. Lee and S.J. Na, Proc. Inst. Mech. Eng. B: J. Eng. Manuf. 209 (1995) 153.

[8] J.J. Lowke, R. Morrow and J. Haidar, J. Phys. D: Appl. Phys. 30 (1997) 2033.

[9] V.A. Nemchinsky, J. Phys. D: Appl. Phys. 27 (1994) 1433.

[10] J. Hu and H.L. Tsai, Heat. Mass. Transfer. 50 (2007) 833.

[11] J.J. Lowke, P. Kovitya and H.P. Schmidt, J. Phys. D: Appl. Phys. 25 (1992) 1600.

[12] M. Tanaka, H. Terasaki, M. Ushio and J.J. Lowke, Metall. Mater. Trans. A 33 (2002) 2043.

[13] C.S. Wu and J.Q. Gao, J. Mater. Sci. Technol. 18 (2002) 43.

[14] G. Xu, J. Hu, and H.L. Tsai, J. Appl. Phys. 104 (2008) 103301-9.

[15] W. Zhang, C.H. Kim and T. DebRoy, J. Appl. Phys. 95 (2004) 5210.

[16] P.F. Mendez, M.A. Ramirez, G. Trapaga and T.W. Eagar, Metall. Mater. Trans. B 32 (2001) 547.

[17] W.H. Kim, H. G. Fan and S. J. Na, Metall. Mater. Trans. B 28 (1997) 679.

[18] H.G. Fan, S-J Na and Y. W. Shi, J. Phys. D: Appl. Phys. 30 (1997) 94.

[19] G.L. Liang, G. Zhou and S.Q. Yuan, Mater. Sci. Eng. A. 499 (2009) 93.

[20] C. Delalondre and O. Simonin, J. Phys. Coll. 51(C5) (1990) 199.

[21] S.V. Patanka, Numerical Heat Transfer and Fluid Flow, McGraw-Hill, New York, 1980, p.126.

[22] Z.H. Chen, Magnesium Alloy, Chemical Industry Press, Beijing, 2005, p. 10. (in Chinese) 